\title{
Property Analysis of Riccati Difference Equation for Load Frequency Controller of Time Delayed Power System Using IMMKF
}

\author{
M. Sumathy ${ }^{1}{ }^{*}$, Adem Kilicman ${ }^{2}$, M. Maria Susai Manuel ${ }^{3}$ and Jesintha Mary ${ }^{4}$ \\ ${ }^{1,3}$ Department of Science and Humanities, R. M. D. Engineering College, \\ Kavaraipettai - 601 206, Tamil Nadu, S. India \\ ${ }^{2}$ Department of Mathematics and Institute for Mathematical Research, \\ Universiti Putra Malaysia, 43400 UPM, Serdang, Selangor, Malaysia \\ ${ }^{4}$ Department of E.E.E, R. M. K. College of Engineering and Technology, \\ Puduvoyal- 601 206, Tamil Nadu, S. India \\ ks.snh@rmd.ac.in ${ }^{*}$,akilic@upm.edu.my \\ hodsnh@rmd.ac.in ${ }^{3}$, jesintha82eee@gmail.com ${ }^{4}$
}

\begin{abstract}
In this paper, initially a mathematical model is formulated for transient frequency of power system considering time delays which occur while transmitting the control signals in open communication infrastructure. Time delay negligence in a power system leads to improper measurement of frequency variation in power system. The study of impact of time delays on the stability of power system is performed by estimating the decay rate of frequency wave form using Kalman Filter (KF). In power system, there is a possibility of multiple time delays. This paper also focusses on developing Interacting Multiple Model(IMM) Algorithm with multiple model space using Kalman Filter(KF) as state estimator tool. The multiple time delays in power system is considered as multiple model space The result shows that KF provides better estimate of correct model for a particular inputset. The qualitative properties of Riccati difference equation(RDE) in terms of state error covariance of IMMKF are also analyzed and presented.
\end{abstract}

Key words: Riccati Difference equations, Power System Stability, Interacting Multiple Model Kalman Filter, Load frequency controller, Time Delays.

AMS Classification: 39A10, 39A30, 39A60, 93C80.

\section{$1 \quad$ Introduction}

In many industrial applications, direct measurement of the state variables is impossible which required state estimation based on the output in the course of the process of state controller model $[1,3]$. Among the various filters used, Kalman filter is incorporated in several applications to 
evaluate the state for power system, signal processing and navigation [3,5] systems with measurement noises. This filter maintains an optimal recursive solution for the least square state estimation problem and its properties including stability. The Riccati equation and its variation, the Matrix Riccati equation appear in numerous scientific and engineering applications such as optimal control and filtering problems [10,20] which plays an important role in the stability analysis of different variations of the Kalman filter. The results are later extended to the non monotonic case [9].

The properties of the solutions of the Riccati equation convergence like monotonicity and stabilizability were discussed $[6,7,8,12]$ by selecting appropriate initial covariance matrix through the monotonic properties $[11,20,24]$. These assumptions were then composed of detectability and stabilizability by for continuous time systems [27] and for discrete-time systems [8]. Interrelated results were acknowledged [3] for time-varying detectable and stabilizable systems. The detailed relations between various Riccati equations and the closed-loop stability of linear quadratic optimal control and estimation are illustrated in $[6,7,12]$.

Power system is one of the most intricate system in the eminent field of engineering theory. Enormous literature is available on the frequency measurement techniques for power system. Frequency is significantly determined by active power whereas the voltage is determined by the reactive power. The obstacles in power system control are the frequency(active power)control and the voltage(reactive power) regulation. The active power control with frequency control is also known as the Automatic Load Frequency Control (ALFC). Time delays in voltage and frequency play a vital role in evaluating amplitude, phase of voltage and current signals. Time-delays in communication between a controller and generators are one of the eminent impediments in stability. Minimal research has been performed in the field of power system stability due to time delay. In order to maintain the stability of power system, multifarious control loops are required. Proper control and maintenance of the frequency stability of a power system requires primary, secondary and tertiary frequency control loops. Generally, Primary Frequency Control (PFC)loop is important for intercepting the frequency decline before triggering the under/over frequency protection relays. The PFC is performed by the governor droop resulting in the steady state errors.

The secondary frequency control (Load Frequency control (LFC) ) [4, 22] is used to regulate the frequency in power systems into a desirable range and to control the interchange power between the different control areas through major tie-lines. Finally, tertiary control level is resending the 
generated units and secondary reserve after a distinct disturbance. An LFC model to consider the effects of frequency control loops for two-area power systems is already developed [13].

The main objective of LFC during load sharing is to restore the balance between load and generation in every control area under definite limits. The delay dependent stability of power system is analyzed using frequency domain approach in $[15,16,17]$. The delay dependent stability of the LFC scheme by using the Lyapunov-theory based delay-dependent criterion and Linear Matrix Inequalities (LMIs) techniques was earlier investigated [19]. The wide range of communication networks in power system control causes unavoidable time delays. The impact of these delays on the stability of one-area and two-area LFC systems and an analytical method to determine delay margins, for stability is proposed in [23]. An LFC approach based on LMI theory to design a robust controller in delayed control signals is proposed in [2] and of the equivalent seven stability criteria is established in [25]. The present estimation problem is based on the measured data to approximate the values of the unknown parameters used from the measured data.

\section{Motivation and Contribution}

Extended Kalman Filter has been applied for estimating various parameters in PFC [21], whereas there is no report in literature on the tracking and estimation of frequency decay in Secondary Frequency Controller (SFC) with this kind of deriving mathematical model and discretization of the system. This paper focuses on the application of an IMMKF for estimating the decay rate in the transient frequency of power system by considering various time delays as different models. This helps as a guide to analyze the impact of time delays on powers system.

The current research article is organized as follows. In section 2, the model of power system incorporated with communication delay is developed and discussed and also the mathematical formulation for transient frequency variation of power system with time delay is presented. Section 3 proposes an IMMKF algorithm for estimating the state of the system and the evaluation of the properties of the Riccati Difference equation through state error covariance is also presented. A detailed discussion on the IMMKF algorithm and the Properties of the Riccati Difference equation such as monotonicity and stability are stated in Section 4. Results of estimating the measurement vector using Kalman filter and identifying the correct input set in multiple model space using IMMKF is presented in section 5. Conclusions are drawn in Section 6.

\section{Power system and its mathematical formulation}

\subsection{Model of Power system}

An illustration of the model with the components of the ALFC loop in power system considering 
the limitations of communication network is shown in Figure1.One of the main limitations in communication network is the time delay arising during the transmission of control signals. This consists of governor, turbine and load generator which are modeled by a transfer function of first order. Multiple factors contribute to the time delay in LFC system. All these delays are appended as a single delay denoted by $e^{-s \tau}$, where $\tau$ is the time delay. The Transfer function of governor $G_{H}$ is $\frac{1}{1+s T_{g i}}$ where $T_{g i}$ is a governor time constant $(\mathrm{s})$, the Transfer function of non reheat turbine $G_{T}$ is $\frac{1}{1+s T_{t i}}$ where $T_{t i}$ is a turbine time constant (s), the Transfer function of PI controller is $k_{p}+\frac{k_{i}}{s}$ and finally the Transfer function of Generator $G_{P}$ is $\frac{K_{p}}{1+s T_{p}}$.

$\Delta f(s)$ is the frequency deviation which acts as a output signal.

Let $\Delta P_{r e f}(s)=k_{p}+\frac{k_{i}}{s} \cdot \Delta f(s)$

is the reference set power which acts as a control input.

Here, $\Delta f(s)$ is fed to PI controller which controls the speed changer position.

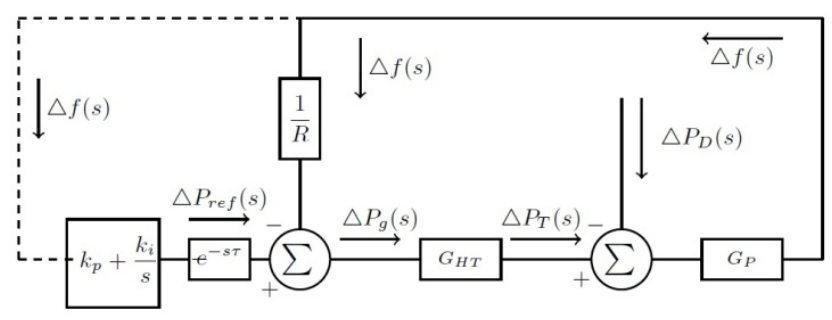

Figure 1. Block diagram of Load Frequency Control

Here $k_{p}, k_{i}$ are the proportional and integral gain constant of the controller and $\mathrm{K}_{p}, T_{p}$ are Gain constant and Time constant for generator.

From the Figure 1, $\Delta f(s)$ can be written as

$\Delta f(s)=\frac{G_{p}}{1+\left[\left(k_{p}+\frac{k_{i}}{s}\right) e^{-s \tau}\right] G_{H T} G_{p}+\frac{1}{R} G_{H T} G_{p}} \cdot \Delta P_{D}(s)$

Here, $G_{H T}=1, G_{p}=\frac{K_{p}}{1+s T_{p}}$ and $\Delta P_{D}(s)=\frac{M}{s}$, where $M$ is the magnitude of step change in load.

Then,$\Delta f(s)=\frac{\frac{K p}{1+s T_{p}}}{1+\left[k_{p}+\frac{k_{i}}{s}\right] \frac{K_{p}}{1+s T p} e^{-s \tau}+\frac{1}{R}\left[\frac{K_{p}}{1+s T p}\right]} \cdot \frac{M}{s}$

Substituting $e^{-s \tau}$ as $\frac{1-s h}{1+s h}$ by Rekasius substitution and after simplification we get, 


$$
\Delta f(s)=\frac{R K_{p}(1+s h) M}{s^{3}+J_{2} s^{2}+J_{1} s+J_{0}}
$$

where $\quad J_{2}=\frac{R h+R T_{p}-R h k_{p} \beta K_{p}+h K_{p}}{R T_{p} h}$

$$
\begin{aligned}
& J_{1}=\frac{R-R h k_{i} \beta K_{p}+R k_{p} \beta K_{p}+K_{p}}{R T_{p} h} \\
& J_{0}=\frac{k_{i} \beta K_{p}}{T_{p} h}
\end{aligned}
$$

Inverse Laplace transformation of $\Delta f(s)$ yields the answer in the form

$f(t)=a_{1} e^{-\alpha_{1} t}+a_{2} e^{-\alpha_{2} t} \cos \omega t+a_{3} e^{-\alpha_{2} t} \sin \omega t$

\subsection{Mathematical Formulation of Transient Frequency Deviation}

The mathematical model for the transient frequency variation of power system is formulated as

$f(t)=a_{1} e^{-\alpha_{1} t}+a_{2} e^{-\alpha_{2} t} \cos \omega t+a_{3} e^{-\alpha_{2} t} \sin \omega t$

The above can be expressed as $f(t)=y_{1}(t)+y_{2}(t)$

where $y_{1}(t)=a_{1} e^{-\alpha_{1} t}$, (exponentially decaying component)

$y_{2}(t)=a_{2} e^{-\alpha_{2} t} \cos \omega t+a_{3} e^{-\alpha_{2} t} \sin \omega t$. (an oscillatory component)

$y_{1}(t)$ and $y_{2}(t)$ are the solutions of the following differential equations

$\dot{y}_{1}=\alpha_{1} y_{1}$.

$\ddot{y}_{2}=-2 \alpha_{2} \dot{y}_{2}-\left(\alpha_{2}{ }^{2}+\omega^{2}\right) y_{2}$.

Using state-space representation, we get

$x_{1}=y_{1}$

$x_{2}=y_{2}$

$\dot{x}_{2}=x_{3}$.

With the definitions of the equations (3.5) - (3.7), equations (3.3) and (3.4) can be rewritten as

$\dot{x}_{1}=-\alpha_{1} x_{1}$

$\dot{x}_{2}=x_{3}$,

$\dot{x}_{3}=-2 \alpha_{2} x_{3}-\left(\alpha_{2}{ }^{2}+\omega^{2}\right) x_{2}$.

Kalman filter is used to estimate the states $x_{1}, x_{2}$ and $x_{3}$ and the parameters $a_{1}, a_{2}, \alpha_{1}, \alpha_{2}, \omega$. These parameters are evaluated as additional states invariant with time. Using Forward Euler discretization method, the augmented state equations is given as,

$x_{1}(k+1)=\left(1-x_{4}(k) \Delta t\right) x_{1}(k)$, 


$$
\begin{aligned}
& x_{2}(k+1)=x_{2}(k)+x_{3}(k) \Delta t, \\
& x_{3}(k+1)=\left(1-2 x_{5}(k) \Delta t\right) x_{3}(k)-x_{6}(k) x_{2}(k) \Delta t, \\
& x_{4}(k+1)=x_{4}(k) \\
& x_{5}(k+1)=x_{5}(k) \\
& x_{6}(k+1)=x_{6}(k)
\end{aligned}
$$

where $x_{4}=\alpha_{1}, x_{5}=\alpha_{2}$ and $x_{6}=\alpha_{2}{ }^{2}+\omega^{2}$.

Here, $k$ is the sampling instance and $\Delta t$ is the sampling interval. The general form of the above equations is

$x(k+1)=f(x(k), u(k), k)+\omega(k)$

The measurement vector $z(k)$ is given by

$$
z(k)=h(x(k), u(k), k)+r(k)
$$

The coefficient matrix $\check{F}, \check{H}$ are the Jacobian matrices are as follows;

$$
\begin{aligned}
& \check{F}=\left.\frac{\partial f(x(k), k)}{\partial x(k)}\right|_{\hat{x}(k)} \\
& =\left[\begin{array}{cccccc}
1-x_{4}(k) \Delta t & 0 & 0 & -x_{1}(k) \Delta t & 0 & 0 \\
0 & 1 & \Delta t & 0 & 0 & 0 \\
0 & -x_{6}(k) \Delta t & 1-2 x_{5}(k) \Delta t & 0 & -2 x_{3}(k) \Delta t & -x_{2}(k) \Delta t \\
0 & 0 & 0 & 1 & 0 & 0 \\
0 & 0 & 0 & 0 & 1 & 0 \\
0 & 0 & 0 & 0 & 0 & 1
\end{array}\right] \\
& \widetilde{H}=\left.\frac{\partial z(x(k), k)}{\partial x(k)}\right|_{\widehat{x}(k)} \\
& =\left[\begin{array}{llllll}
1 & 1 & 0 & 0 & 0 & 0
\end{array}\right]
\end{aligned}
$$

In the next section, the estimation of state variables of the system with the help of Kalman filter is discussed and the development of interacting multiple model is presented. 


\section{The IMMKF algorithm}

The IMM algorithm incorporates $r$ interacting filters functioning simultaneously with every filter corresponding to a model of the following stochastic hybrid system [5]

$x(\bar{k})=\breve{F}_{m(k)} x(\bar{k}-1)+\omega_{m(k)}(\bar{k})$

$\check{z}(\bar{k})=\breve{H}_{m(k)} x(\bar{k})+v_{m(k)}(\bar{k})$

where $x \in \Re^{n}$ is the state of every system and $\check{z} \in \mathfrak{R}^{P}$ is the measurement vector where

$$
E\left\{\left[\begin{array}{c}
\omega_{i} \\
v_{i}
\end{array}\right]\left[\begin{array}{ll}
\omega_{j}^{T} & v_{j}^{T}
\end{array}\right]\right\}=\left[\begin{array}{cc}
\check{Q} & 0 \\
0 & \check{R}
\end{array}\right] \delta_{i j}
$$

with $\check{Q}$ non-negative $(\check{Q} \geq 0), \check{R}$ positive definite $(\check{R}>0)$ and $\delta_{i j}$ is the Kronecker delta. By factoring $\breve{Q}$ and $\breve{R}$ has $\breve{R}=\left(\breve{R}^{1 / 2}\right)\left(\breve{R}^{1 / 2}\right)^{T}$ and $\breve{Q}=L L^{T} . \breve{F}_{m(k)}$ and $\breve{H}_{m(k)}$ are the matrices of the system corresponding to every model $m(k) \epsilon\{1,2 \ldots r\}$ at time $\bar{k}: \omega_{m(k)}(\bar{k})$ and $v_{m(k)}(\bar{k})$ are uncorrelated Gaussian noise vectors with white zero mean, $\check{Q}_{m(k)}$ and $\breve{R}_{m(k)}$ are the respective covariance matrices. The evolvement of the model $m(k)$ is denoted by $p[m(k)=\hat{\jmath} \mid m(k-1)=\hat{\imath}]=\pi_{\hat{\imath} \hat{\jmath}}$ for $\hat{\imath}, \hat{\jmath}=1,2 \ldots r$, where $\pi_{\hat{\imath} \hat{\jmath}}$ is a constant $; p[. \mid$.$] symbolize a$ conditional probability. In the above system, for all $\hat{\imath}, \hat{\jmath}=1,2 \ldots r$, we consider that $\breve{F}_{\hat{\jmath}}$ is nonsingular with $0<\xi_{1} I \leq \check{Q}_{\hat{\jmath}} \leq \xi_{2} I, 0<\xi_{3} I \leq \check{R}_{\hat{\jmath}} \leq \xi_{4} I$ where $\xi_{1}, \xi_{2}, \xi_{3}, \xi_{4}$ are inverses of the maximum acceptable values by Bryson's rule and $I$ is the identity matrix.

Let $Z^{\bar{k}}:=\{\check{z}(1), \check{z}(2), \ldots . . \check{z}(\bar{k})\}$ be the set of measurements up to time $\bar{k}$. The IMM algorithm calculates the relative posterior mean $\hat{x}_{\hat{\jmath}}(\bar{k})$ and $\mathcal{P}_{\hat{\jmath}}(\bar{k})$ for each Kalman filter $\hat{\jmath}$, and the mode probability $\alpha_{\hat{\jmath}}(\bar{k}):=p\left[m(k)=\hat{\jmath} \mid Z^{k}\right]$. Let us suppose that, $\alpha_{\hat{\jmath}}(\bar{k}-1), \hat{x}_{\hat{\jmath}}(\bar{k}-1)$, and $\mathcal{P}_{\hat{\jmath}}(\bar{k}-$ 1) for $\hat{\jmath}=1,2 \ldots r$ are estimated from the last iteration at time $\bar{k}-1$, then the IMM algorithm computes the following for every $\bar{k}$ :

\section{- Interaction /Mixing}

Calculate the mixing probability

$\gamma_{\hat{\jmath} \hat{\imath}}(\bar{k}-1):=p\left[m(k-1)=\hat{\imath} \mid m(k)=\hat{\jmath}, Z^{\bar{k}-1}\right]=\frac{1}{\sum_{l=1}^{r} \pi_{l \hat{\jmath}} \alpha_{l}(\bar{k}-1)} \pi_{\hat{\imath} \hat{\jmath}} \alpha_{\hat{\imath}}(\bar{k}-1)$

For every Kalman filter $\hat{\jmath}$ the initial conditions are calculated as

$$
\begin{aligned}
& \hat{x}_{\hat{\jmath} 0}(\bar{k}-1)=\sum_{\hat{\imath}=1}^{r} \gamma_{\hat{\jmath} \hat{\imath}}(\bar{k}-1) \hat{x}_{\hat{\imath}}(\bar{k}-1), \\
& \mathcal{P}_{\hat{\jmath} 0}(\bar{k}-1)=\sum_{\hat{\imath}=1}^{r}\left\{\mathcal{P}_{\hat{\imath}}(\bar{k}-1)+\left[\widehat{x}_{\hat{\imath}}(\bar{k}-1)-\widehat{x}_{\hat{\jmath} 0}(\bar{k}-1)\right]\left[\widehat{x}_{\hat{\imath}}(\bar{k}-1)-\widehat{x}_{\hat{\jmath} 0}(\bar{k}-1)\right]^{T}\right\} \gamma_{\hat{\jmath} \hat{\imath}}(\bar{k}-1) .
\end{aligned}
$$

\section{- Kalman Filtering}

Compute $\hat{x}_{\hat{\jmath}}(\bar{k})$ and $\mathcal{P}_{\hat{\jmath}}(\bar{k})$ using Kalman Filter for each $\hat{\jmath}$ model. 


$$
\begin{aligned}
& \hat{x}_{\hat{\jmath}}(\bar{k})=\breve{F}_{\hat{\jmath}} \hat{x}_{\hat{\jmath} 0}(\bar{k}-1)+\breve{K}_{\hat{\jmath}}(\bar{k}) v_{\hat{\jmath}}(\bar{k}) \\
& \mathcal{P}_{\hat{\jmath}}(\bar{k} \mid \bar{k}-1)=\left[\breve{F}_{\hat{\jmath}} \mathcal{P}_{\hat{\jmath} 0}(\bar{k}-1) \breve{F}_{\hat{\jmath}}^{T}+\breve{Q}_{\hat{\jmath}}\right] \\
& \mathcal{P}_{\hat{\jmath}}(\bar{k})=\left[\mathcal{P}_{\hat{\jmath}}^{-1}(\bar{k} \mid \bar{k}-1)+\breve{H}_{\hat{\jmath}}^{T} \breve{R}_{\hat{\jmath}}{ }^{-1} \breve{H}_{\hat{\jmath}}\right]^{-1}
\end{aligned}
$$

where $v_{\hat{\jmath}}(\bar{k})=\check{z}(\bar{k})-\breve{H}_{\hat{j}} \breve{F}_{\hat{j}} \hat{x}_{\hat{j} 0}(\bar{k}-1)$ represents the residual, $\breve{K}_{\hat{\jmath}}(\bar{k})$ is the gain of the Kalman filter, and $\mathcal{P}_{\hat{j}}(\bar{k}), \mathcal{P}_{\hat{j}}(\bar{k} \mid \bar{k}-1)$ is the posterior (prior) state covariance.

By defining $\breve{E}=\breve{R}^{-1 / 2} \breve{H}$, we can rewrite the Riccati equation in the normalized form $\mathcal{P}_{\hat{\jmath}}(\bar{k})=\breve{F}_{\bar{k}} \mathcal{P}_{\hat{\jmath}}(\bar{k} \mid \bar{k}-1) \breve{F}_{\bar{k}}{ }^{T}-\breve{F}_{\bar{k}} \mathcal{P}_{\hat{\jmath}}(\bar{k} \mid \bar{k}-1) \breve{H}_{\bar{k}}{ }^{T}\left(\breve{H}_{\bar{k}} \mathcal{P}_{\hat{\jmath}}(\bar{k} \mid \bar{k}-1) \breve{H}_{\bar{k}}{ }^{T}+I\right)^{-1} \breve{H}_{\bar{k}} \mathcal{P}_{\hat{\jmath}}(\bar{k} \mid \bar{k}-$

1) $\breve{F}_{\bar{k}}^{T}+L L^{T}$

\section{- Model Probability Update}

Herewith $\Lambda_{\hat{\jmath}}(\bar{k}):=N_{p}\left(v_{\hat{\jmath}}(\bar{k}) ; 0 ; S_{\hat{\jmath}}(\bar{k})\right)$ is the Likelihood function with $p$ dimension and $v_{\hat{\jmath}}(\bar{k}) ; N_{p}(. ; 0 ; \Sigma)$ is a $p$-dimensional multivariate Gaussian probability density function along with mean zero and $\Sigma$ covariance and $S_{\hat{\jmath}}(\bar{k})$ denotes the residual covariance. The model probability is then given by $\alpha_{\hat{\jmath}}(\bar{k})=\frac{1}{\sum_{l=1}^{r} \bar{\Lambda}_{l}(\bar{k}) \alpha_{\bar{l}}(\bar{k})} \Lambda_{\hat{\jmath}}(\bar{k})\left(\sum_{\hat{\imath}=1}^{r} \pi_{\hat{\imath} \hat{\jmath}} \alpha_{\hat{\imath}}(\bar{k}-1)\right)$.

\section{- State Estimate and Covariance combiner}

The state estimator and the covariance combiner are respectively calculated as

$\hat{x}(\bar{k})=\sum_{\hat{\jmath}=1}^{r} \alpha_{\hat{\jmath}}(\bar{k}) \hat{x}_{\hat{\jmath}}(\bar{k})$.

$\mathcal{P}(\bar{k})=\sum_{\hat{\jmath}=1}^{r}\left\{\mathcal{P}_{\hat{\jmath}}(\bar{k})+\left[\hat{x}_{\hat{\jmath}}(\bar{k})-\hat{x}(\bar{k})\right]\left[\hat{x}_{\hat{\jmath}}(\bar{k})-\hat{x}(\bar{k})\right]^{T}\right\} \alpha_{\hat{\jmath}}(\bar{k})$.

\section{Properties of Riccati Difference Equation}

$$
\begin{aligned}
& x(\bar{k})=\breve{F}_{m(k)} x(\bar{k}-1)+\omega_{m(k)}(\bar{k}) \\
& \check{z}(\bar{k})=\breve{H}_{m(k)} x(\bar{k})+v_{m(k)}(\bar{k})
\end{aligned}
$$

where $x \in \Re^{n}$ is the state of every system and $\check{z} \in \Re^{P}$ is the measurement vector.

This section is concerned with the stability and monotonicity properties of solutions $\{\mathcal{P}(\bar{k})\}$ of the Riccati Difference Equation (RDE) of optimal filtering [6,7]. The associated Riccati Difference Equation is

$$
\mathcal{P}(\bar{k}+1)=\breve{F} \mathcal{P}(\bar{k}) \breve{F}^{T}-\breve{F} \mathcal{P}(\bar{k}) \breve{H}^{T}\left(\breve{H} \mathcal{P}(\bar{k}) \breve{H}^{T}+\breve{R}\right)^{-1} \breve{H} \mathcal{P}(\bar{k}) \breve{F}^{T}+\breve{Q} .
$$

To obtain the results of monotonicity and stability in this section, the following fundamental theorems are needed.

\subsection{Monotonicity}

The subsequent theorems characterize the monotonic property of the solution of the RDE, which performs an important features in the stability analysis of optimal filters. 
Theorem 1.[7] Let $\mathcal{P}_{1}(\bar{k})$ and $\mathcal{P}_{2}(\bar{k})$ are the solutions of two RDEs with the matrices $\breve{F}, \breve{H}$ and $\breve{R}$ matrices with initial conditions $\mathcal{P}_{1}(0)=\mathcal{P}_{1} \geq 0$ and $\mathcal{P}_{2}(0)=\mathcal{P}_{2} \geq 0$ with $t \geq 0$ and for all $\bar{k} \geq 0$ are

$\mathcal{P}(t+\bar{k})=\breve{F} \mathcal{P}(t+\bar{k}-1) \breve{F}^{T}-\breve{F} \mathcal{P}(t+\bar{k}-1) \breve{H}^{T}\left(\breve{H} \mathcal{P}(t+\bar{k}-1) \breve{H}^{T}+\check{R}\right)^{-1} \breve{H} \mathcal{P}(t+\bar{k}-$ 1) $\check{F}^{T}+\check{Q}$

$\mathcal{P}(t+\bar{k}+1)=\breve{F} \mathcal{P}(t+\bar{k}) \breve{F}^{T}-\breve{F} \mathcal{P}(t+\bar{k}) \breve{H}^{T}\left(\breve{H} \mathcal{P}(t+\bar{k}) \breve{H}^{T}+\check{R}\right)^{-1} \breve{H} \mathcal{P}(t+\bar{k}) \breve{F}^{T}+\check{Q}$,

and have the two solutions $\mathcal{P}(t+\bar{k}) \geq 0$ and $\mathcal{P}(t+\bar{k}-1) \geq 0$.

Then, $\mathcal{P}(t+\bar{k}+1)=\breve{F}(t+\bar{k}+1) \mathcal{P}(t+\bar{k}) \breve{F}^{T}(t+\bar{k}+1)-\breve{F}(t+\bar{k}+1) \mathcal{P}(t+\bar{k}) \breve{H}^{T}$

$$
\left(\breve{H} \mathcal{P}(t+\bar{k}) \breve{H}^{T}+\check{R}(\bar{k})\right)^{-1} \breve{H} \mathcal{P}(t+\bar{k}) \breve{F}^{T}(t+\bar{k}+1) .
$$

where $\breve{F}(t+\bar{k}+1)=\breve{F}-\breve{F} \mathcal{P}(t+\bar{k}+1) \breve{H}^{T}\left(\breve{H} \mathcal{P}(t+\bar{k}+1) \breve{H}^{T}+\breve{R}(t+\bar{k}+1)\right)^{-1} \breve{H}$ and $\breve{R}(t+\bar{k})=\breve{H} \mathcal{P}(t+\bar{k}+1) \breve{H}^{T}+\check{R}$.

Theorem 2. Suppose, that the solution of the RDE (5.6) has a solution $\mathcal{P}(t+\bar{k}) \leq 0$ for all $t \geq$ 0 and $\bar{k}=0$. Then, $\mathcal{P}(\bar{k})$ is monotonically non increasing for all subsequent time $\mathcal{P}(t+\bar{k}) \leq \mathcal{P}(t+\bar{k}+1)$ for all $\bar{k} \geq 0$.

Theorem 3. Suppose, that the solution of the RDE (5.6) has a solution $\mathcal{P}(t+\bar{k}) \geq 0$ for all $t \geq$ 0 and $\bar{k}=0$. Then, $\mathcal{P}(\bar{k})$ is monotonically non increasing for all subsequent time $\mathcal{P}(t+\bar{k}) \geq \mathcal{P}(t+\bar{k}+1)$ for all $\bar{k} \geq 0$.

Let us also note that, $\{\mathcal{P}(\bar{k})\}$ which satisfy the RDE is a monotonic non increasing sequence, then $\breve{Q}(\bar{k})$ is not necessarily monotonic sequence of non negative definite matrices defined by

$\check{Q}(\bar{k})=\mathcal{P}(\bar{k})-\breve{F} \mathcal{P}(\bar{k}) \check{F}^{T}+\check{F} \mathcal{P}(\bar{k}) \check{E}^{T}\left(\check{E} \mathcal{P}(\bar{k}) \check{E}^{T}+\check{R}\right)^{-1} \check{E} \mathcal{P}(\bar{k}) \check{F}^{T}$

satisfying $\check{Q}(\bar{k}) \geq L L^{T}$ and also $\check{Q}(\bar{k}) \geq 0$

\subsection{Stability}

By factoring $\breve{Q}$ and $\breve{R}$ has $\breve{R}=\left(\breve{R}^{1 / 2}\right)\left(\breve{R}^{1 / 2}\right)^{T}$ and $\check{Q}=L L^{T}$ and by defining $\breve{E}=\check{R}^{-1 / 2} \breve{H}$ we can rewrite the Riccati equation in a normalized form as

$\mathcal{P}(\bar{k}+1)=\breve{F} \mathcal{P}(\bar{k}) \breve{F}^{T}-\breve{F} \mathcal{P}(\bar{k}) \breve{E}^{T}\left(\breve{E} \mathcal{P}(\bar{k}) \breve{E}^{T}+I\right)^{-1} \breve{E} \mathcal{P}(\bar{k}) \breve{F}^{T}+L L^{T}$

The closed -loop state transition matrix of the Kalman filter is

$\breve{F}(\bar{k})=\breve{F}-\breve{F} \mathcal{P}(\bar{k}) \breve{E}^{T}\left(\breve{E} \mathcal{P}(\bar{k}) \check{E}^{T}+I\right)^{-1} \breve{E}=\breve{F}-\breve{K}(\bar{k}) \check{E}$.

\section{Theorem 4}

Consider the $\operatorname{RDE}$ (5.10) with initial condition $\mathcal{P}_{0}$ and solution $\mathcal{P}(\bar{k})$. Define the sequence of 
matrices $\check{Q}(\bar{k})$ by eqn.(5.9). If

1. $[\breve{E}, \breve{F}]$ is detectable.

2. $[\breve{F}, L]$ is stabilizable.

3. $\mathcal{P}_{0} \geq 0$ is such that $\check{Q}_{0} \geq L L^{T}$,

then the solution sequence $\{\mathcal{P}(\bar{k})\}$ of the RDE is stabilizing for all $\bar{k} \geq 0$, i.e $\left|\lambda_{i}(\breve{F}(\bar{k}))\right|<1$ for all $\bar{k} \geq 0$ and for $i=1,2, \ldots . n$, with $\breve{F}(\bar{k})$ denoted by eqn. (5.8) and $\lambda_{i}($.$) denoting the$ individual eigen values.

\section{Theorem 5}

Consider the RDE (5.10). Define $\check{Q}_{0}$ as in eqn. (5.9). If

1. $[\breve{E}, \breve{F}]$ is detectable.

2. $\left[\breve{F}, \check{Q}_{0}{ }^{1 / 2}\right]$ is stabilizable.

3. $\mathcal{P}_{0} \geq 0$ is such that $\check{Q}_{0} \geq L L^{T}$, then the solution sequence $\{\mathcal{P}(\bar{k})\}$ with initial condition $\mathcal{P}_{0}$ is stabilizing for each $\bar{k}$.

To conclude that, a real symmetric nonnegative definite solution $\mathcal{P}^{*}$ of the RDE is called a strong solution if the corresponding state transition matrix $\breve{F} \triangleq \breve{F}-\breve{F} \mathcal{P}^{*} \breve{E}^{T}\left(\breve{E} \mathcal{P}^{*} \breve{E}^{T}+I\right)^{-1} \breve{E}$ has all its eigen values inside or on the unit circle and hence it is stabilizing and by comparing the RDE (5.10) with eqn. (5.9) we have $\breve{Q}(\bar{k})=L L^{T}+\mathcal{P}(\bar{k})-\mathcal{P}(\bar{k}+1)$, which leads to monotonicity.

\section{Results and Observations}

In this paper, Kalman filter is introduced as a tool to estimate the state variables of power system. The Multiple Kalman filter is in parallel comparing how well each Kalman Filter matches true measurement from the actual measurements of system. Implementing the IMM allows for an arbitrary number of Kalman Filters to be operated each one modeling a different point with different time delays. Although number of Kalman Filters in IMM framework is conceptually unlimited, the computational requirement of IMM increase linearly as more models are evaluated. In this section, the mathematical formulation of IMM is established. The parameters that define IMM and the results of IMM is presented with KF as the estimator of individual models.Although IMM was developed as a tool for target tracking environment, the basic formula can also be applied to the estimation of impact of time delay on the frequency waveform of power system. The set of time delayed power system models are defined as the model space in IMM. Each model is defined by the decay rate $\left(\alpha_{1}\right)$ of exponential component and decay rate $\left(\alpha_{2}\right)$ of oscillatory component which indirectly related to the study of impact of time delays on the frequency wave form of power system. 
In this research, model space consists of 2 models. These models are chosen in order to mean the power system is subjected to different time delays while transmitting the control signals in LFC system. The operation of KF as the estimation for IMM is considered.

To demonstrate the application of IMMKF algorithm for the estimation of frequency decay in Power system, a single area LFC system is considered. The corresponding nomenclature is given in Appendix A. The parameters of the system [16] are as follows;

$\mathrm{R}=2, \mathrm{~T}_{\mathrm{p}}=20, \mathrm{M}=0.01, k_{\mathrm{p}}=0.1, K_{p}=0.1, \beta=0.51, \mathrm{~h}=5$

Substituting these values in eqn. (2.4),

$\Delta f(s)=\frac{0.02(1+5 s)}{s^{3}+0.2724 s^{2}+0.01296 s+0.00051}$

where $a_{1}=-0.05908, a_{2}=0.05908, a_{3}=2.1356, \alpha_{1}=0.22491, \alpha_{2}=0.02377$ and $\omega=$ 0.04126 .

The parameters identified as state variables in the system are

$x_{1}(k+1)=\left(1-x_{4}(k) \Delta t\right) x_{1}(k)$,

$x_{2}(k+1)=x_{2}(k)+x_{3}(k) \Delta t$,

$x_{3}(k+1)=\left(1-2 x_{5}(k) \Delta t\right) x_{3}(k)-x_{6}(k) x_{2}(k) \Delta t$,

$x_{4}(k+1)=x_{4}(k)$

$x_{5}(k+1)=x_{5}(k)$

$x_{6}(k+1)=x_{6}(k)$

The initial values assigned for the elements of initial state vector $\hat{X}=\left[\begin{array}{llllll}x_{1} & x_{2} & x_{3} & x_{4} & x_{5} & x_{6}\end{array}\right]^{T}$ is derived from eqn.(2.5) and the initial values for $\mathrm{h}=5 \mathrm{~s}$ is calculated as $\left[\begin{array}{lll}-0.05908 & 0.05908\end{array}\right.$ $\left.\begin{array}{llll}0.08611 & 0.22491 & 0.02377 & 0.002267\end{array}\right]^{\mathrm{T}}$.

The initial covariance matrix , which is of the form of $(6 \times 6)$ matrix is assumed to have all elements as 15 and the noise vector $\check{Q}$ is given a value of 0.05 and $\check{R}$ as 0.001 . The coefficient matrix $\breve{F}, \breve{H}$ are the Jacobian matrices are as follows

$$
\begin{aligned}
& \breve{F}=\left[\begin{array}{cccccc}
0.9775 & 0 & 0 & 0.0059 & 0 & 0 \\
0 & 1 & 0.1 & 0 & 0 & 0 \\
0 & -0.00020 .9952 & 0 & -0.0172 & -0.0059 \\
0 & 0 & 0 & 1 & 0 & 0 \\
0 & 0 & 0 & 0 & 1 & 0 \\
0 & 0 & 0 & 0 & 0 & 1
\end{array}\right] \\
& \breve{H}=\left[\begin{array}{llllll}
1 & 1 & 0 & 0 & 0 & 0
\end{array}\right] .
\end{aligned}
$$

For different $h$ values, the initial values of the state vector are calculated and presented in table1 below.

Table 1: Initial values of the state vector

\begin{tabular}{|l|l|l|l|l|l|}
\hline & $\mathbf{h}=\mathbf{5}$ & $\mathbf{h}=\mathbf{1 0}$ & $\mathbf{h}=\mathbf{1 5}$ & $\mathbf{h}=\mathbf{2 0}$ & $\mathbf{h}=\mathbf{3 5}$ \\
\hline
\end{tabular}




\begin{tabular}{|c|c|c|c|c|c|}
\hline $\boldsymbol{\alpha}_{\mathbf{1}}$ & 0.22491 & 0.14876 & 0.12903 & 0.120229 & 0.11007 \\
\hline $\boldsymbol{\alpha}_{\mathbf{2}}$ & 0.02377 & 0.0118462 & 0.00505 & 0.00111 & -0.00452 \\
\hline $\boldsymbol{\omega}$ & 0.04126 & 0.039246 & 0.03595 & 0.03255 & 0.02533 \\
\hline
\end{tabular}

When $h$ increases the decay rate decreases which means that for the higher value of time delays, the system may go unstable. This is verified through simulation. Initially, Kalman filter is applied for estimating the measurement vector of the system. It is presented in the following figures Figures 2(a)-2(c).

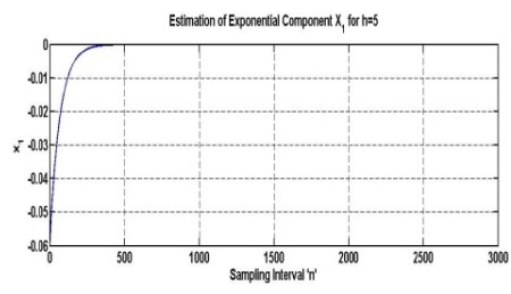

Figure 2(a)

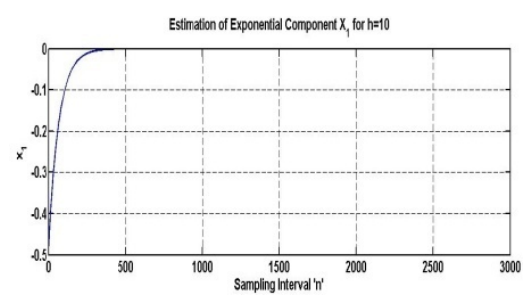

Figure 3(a)

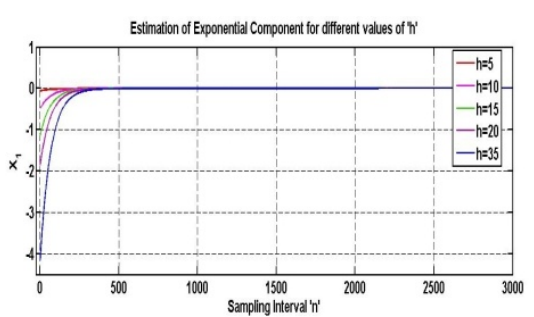

Figure 4(a)

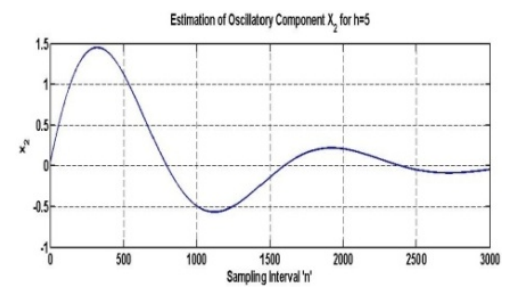

Figure 2(b)

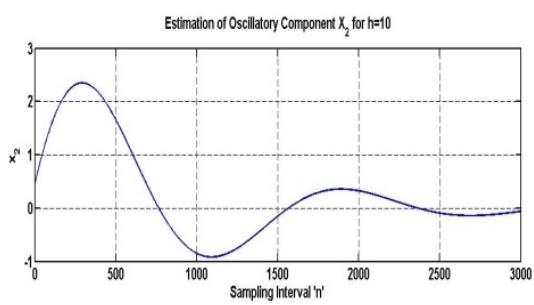

Figure 3(b)

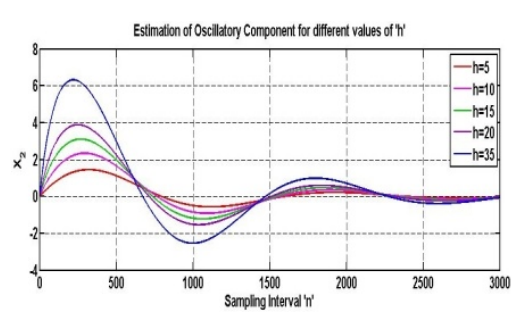

Figure 4(b)

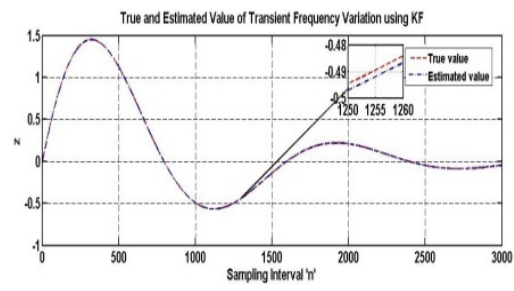

Figure 2(c)

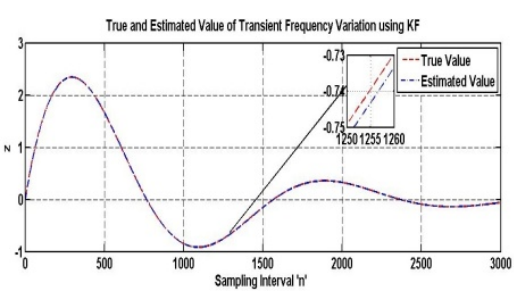

Figure 3(c)

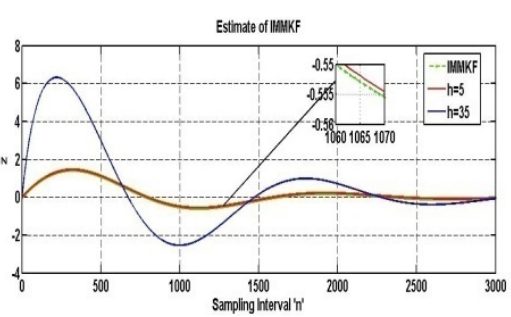

Figure 4(c)

For $h=5 s$, estimation of the state vector $x_{1}$ which is the exponential component presented in Figure 2(a). In Figure 2(b) represents the estimation of the oscillatory component $x_{2}$. Figure 2(c) shows the Frequency deviation at a particular bus following a step load distribution in the power system. This figure is the combination of the exponential component and oscillatory component which is the measurement vector. The figure shows that the Kalman filter is more effective in estimating the frequency decay of the power system. Similarly, for increasing value of the time delay $h=10 \mathrm{~s}$, estimation of the state vectors for exponential and oscillatory components is presented in Figure 3(a) and Figure 3(b). Also, tracking the frequency decay using Kalman Filter is shown in Figure 3(c). For different values of $h$, Figure 4(a) shows exponential component and oscillatory component is presented in Figure 4(b). 
The work is further extended by applying IMMKF by considering two different time delays with $h=5 s$ and $h=35 s$ as two different models. The operation of KF as the estimation for IMM is considered. The result shows that KF provides better estimate of correct model for a particular input set. Figure 4(c) shows the model estimate of IMMKF when the input data set is for $\mathrm{h}=5 \mathrm{~s}$.

The properties of the RDE such as stability and monotonicity are also analyzed and verified.

Using the eqn.(5.7), the solution of $\mathrm{RDE}(5.6)$ is

$$
P^{*}=1.0 e-014 *\left[\begin{array}{cccccc}
0 & 0 & 0 & 0 & 0 & 0 \\
0.0888 & 0.1776 & 0.0888 & 0.0888 & 0.0888 & 0.0888 \\
0.3553 & 0.3553 & 0.1776 & 0.3553 & 0.3553 & 0.3553 \\
0.3553 & 0.3553 & 0 & 0.3553 & 0.3553 & 0.3553 \\
0.3553 & 0.3553 & 0 & 0.3553 & 0.3553 & 0.3553 \\
0.3553 & 0.3553 & 0 & 0.3553 & 0.3553 & 0.3553
\end{array}\right]
$$

Therefore, $P_{0} \geq 0$ and $P_{0} \geq P^{*}$

$P^{*}$ is a strong solution and it is also found that the all the eigen values are inside or on the unit circle. Hence it is stabilizing.

In the same manner, $\check{Q}_{0}=L L^{T}+P_{0}-P_{1}$

Using this it is observed that the state error covariance matrix of Ricatti equation is monotonic in nature.

\section{Conclusion}

In this paper, the transient frequency deviation in the power system following a load disturbance is mathematically formulated. And the Kalman filter was introduced as a means to estimate the decay rate of frequency waveform which indirectly related to the study of impact of time delay on the stability of power system. The application of the IMMKF technique for estimation of frequency decay, in one area LFC of power system with different time delay, is considered and the results of IMM when using KF as a state estimator for each model is also presented. KF provides a better estimate of the correct model. The impact of time delays in power system on the decay rate of frequency variation is also analyzed and presented. The variation of decay rate for various time delays is also shown in results. Moreover, the stability and monotonicity property of Riccati Difference Equation is analyzed.

\section{Appendix A}

\section{Nomenclature}

$\bar{k}$ - time instant.

$x(\bar{k})$ - State Vector at time $\mathrm{k}$. 
$z(\bar{k})$ - Output Vector.

$\omega(\bar{k})$ - Process Noise Vector.

$v(\bar{k})$ - Measurement Noise Vector.

$\breve{F} \quad$ - State Transition Matrix.

$\breve{H} \quad$ - Measurement Matrix.

$\check{Q}$ - Process Noise Covariance Matrix.

$\check{R}$ - Measurement Noise Covariance Matrix.

$\mathcal{P}$ - State Error Covariance Matrix.

$\breve{K}(\bar{k})$ - Kalman Filter Gain.

$\mathrm{p}\{\}-$. Probability.

$\pi_{\hat{\imath} \hat{\jmath}}$ - Model transition probability.

$\Delta P_{r e f}$ - Change in the reference power setting.

$\Delta P_{D}$ - Load disturbance (p.u.MW).

$\Delta f(s)-$ Frequency deviation $(\mathrm{Hz})$.

$\mathrm{K}_{p}-$ Generator gain constant.

$T_{p}$ - Generator time constant (s).

$T_{g i}-$ Governor time constant (s).

$T_{t i}$ - Turbine time constant (s).

R- Speed regulation due to governor action (Hz/p.u MW).

M- Step change in load.

$k_{p}$ - Proportional gain.

$k_{i}$-Integral gain.

\section{References}

[1].Agarwal, R.P.(2000). Difference Equations and Inequalities, MarcelDekker, New York.

[2].Ahmadi,A.,\& Aldeen,.M.( 2016). An LMI approach to the design of robust delay-dependent overlapping load frequency control of uncertain power systems. International Journal of Electrical Power and Energy Systems, 81, 48-63.

[3]. Anderson, B. D. O., \& Moore, J. B.(1979). Optimal Filtering, Prentice Hall, Englewood 
Cliffs, New Jersey.

[4].Ballesteros, F., \& De Arriaga, F.(1989). Optimal control of power systems and the Riccati equation. Applied Mathematics and Computation, 33(1),43-52.

[5].Bar-Shalom, Y., \& Rong Li, X.(1995). Multitarget-MultisensorTracking: Principles and Techniques, YBS Publishing, Storrs, Connecticut.

[6].Bitmead, R. R., Gevers, M., \& Petersen, I.R.(1985). Monotonicity and stabilizabilityproperties of solutions of the Riccati difference equation:Propositions, lemmas, theorems, fallacious conjectures and counter examples.Systems\& Control Letters 5, 309-315.

[7].Bitmead, R. R., \& Gevers, M.(1991). Riccati difference and differential equations: convergence, monotonicity and stability, The Riccati equation, Communications and Control Engineering Series, 263- 291, Springer, Berlin.

[8]. Cai, X., Ding, Y. S. \& Li,S.Y. (2017). Convergent Properties of Riccati Equation with Application to Stability Analysis of State Estimation, Hindawi, Mathematical Problems in Engineering, 1-8.

[9].De Souza, C. E.(1989). On stabilizing properties of solutions of the Riccati difference equation. IEEE Transactions on Automatic Control, 34(12), 1313 - 1316,

[10]. Ferrante, A., \& Ntogramatzidis,L.(2013). The generalised discrete algebraic Riccati equation in linear-quadratic optimal control. Automatica, 49(2), 471-478.

[11].Freiling, G., \& Ionescu, V.(2001). Monotonicity and convexity properties of matrix Riccati equations. IMA Journal of Mathematical Control and Information, 18(1), 61-72.

[12].Grigoryan, G.A.(2001). Properties of solutions of Riccati equation, Journal of Contemporary Mathematical Analysis, 42(4), 184-197.

[13].Hsu,Y.Y.\& Chan, W.C.(1987). Coordinated frequency and voltage control of synchronous generators. IEEE Transactions on Aerospace and Electronic Systems, AES-23, 2-10.

[14]. Ibraheem, \& Prabhat Kumar.(2004). A Novel Approach to the Matrix Riccati Equation Solution: An Application to Optimal Control of Interconnected Power Systems. Electric Power Components and Systems, 32 (1), 33-52.

[15].Jesintha Mary,T., \& Rangarajan,P.(2015). Delay Dependent stability analysis of LFC using Rekasius substitution and resultant theory. International Journal of Applied Engineering Research, 10(10), 25107-25116.

[16].Jesintha Mary,T. \& Rangarajan,P.(2016). Delay Dependent stability analysis of power system considering communication delays. International Transations on Electriacl Energy Systems, 1-13.

[17]. Jesintha Mary,T. (2017). Delay-Dependent stability analysis of LFC in interconnected 
power system using Advanced Clustering with Frequency Sweeping Method. Journal of Advanced Research in Dynamical and Control system, 17, 1259- 1269.

[18].Jia, H., Guangyu, N., Lee, S.T., \& Zhang, P.(2006). Study on the Impact of Time Delay to Power System Small Signal Stability. IEEE Melecon, 62(2), 1011-1014.

[19]. Jiang, L., Yao, W., Wu, H., Wen ,J.Y., \& Cheng, S.J.(2012). Delay dependent stability for load frequency control with constant and time varying delays. IEEE Transactions on Power Systems, 27(2), 932-941.

[20]. Jonathan , A. Q., Jo ao Viana da F. Neto, \& e Allan Kardec Barros.(2015). Solution of Algebraic Riccati Equation for optimal control using Non-Square estimator. IEEE 24th International Symposium on Industrial Electronics (ISIE), 125-130.

[21]. Ramana Reddy, P.(1979). Studies on power system model reduction and control. (Doctoral dissertation). Sri Venkateswara University, India.

[22].Routray, A., Pradhan, A. K.,\& Rao, K.P.(2002). A Novel Kalman filter for frequency estimation of distorted signals in power system. IEEE Transactions On Instrumentation and Measurement, 51(3), 469-79.

[23].Sonmez, S., Ayasun, S., \& Nwankpa, C.(2016). An exact method for computing delay margin for stability of load frequency control systems with constant communication delays. IEEE Transactions on Power Systems, 31(2), 370- 377.

[24]. Sumathy, M., Kilicman, A., Manuel, M. M. S., \& Mary, J. (2019). Qualitative study of Riccati difference equation on maneuvering target tracking and fault diagnosis of wind turbine gearbox. Cogent Engineering, 6(1), 1621423.

[25].Tan,C., Zhang,H.,\& Wong,.W.S. (2017). Delay-Dependent Algebraic Riccati Equation to Stabilization of Networked Control Systems: Continuous-Time Case, IEEE Transactions on Cybernetics, 48(10), 2783- 2794.

[26].Xu, S.Y., \& Lam, J.(2007). On equivalence and efficiency of certain stability criteria for time-delay systems. IEEE Transactions on Automatic Control, 52(1), 95-101.

[27].Zadeh, R. A., Ghosh, A.,\&Ledwich, G.(2010). Combination of Kalman Filter and LeastError Square Techniques in Power System. IEEE Transactions on Power Delivery ,25(4), 2868-2880.

[28].Zheng,J.\& Qiu,.L.(2014). On the Existence of a Mean-Square Stabilizing Solution to a Modified Algebraic Riccati Equation. 19th IFAC World Congress, 24-29. 